\title{
Untargeted Metabolomics Combined with Bioassay Reveals the Change in Critical Bioactive Compounds during the Processing of Qingzhuan Tea
}

\author{
Peng-Cheng Zheng ${ }^{1,2,+} \oplus$, Chun-Yin Qin ${ }^{1,+}$, Pan-Pan Liu ${ }^{2}$, Lin Feng ${ }^{1,2}$, Tie-Jun Ling ${ }^{1}$, Jing-Ming Ning ${ }^{1}$, \\ Liang Zhang ${ }^{1, *}$ and Xiao-Chun Wan ${ }^{1, *}$ \\ 1 State Key Laboratory of Tea Plant Biology and Utilization, Anhui Agricultural University, \\ Hefei 230036, China; zpct15@163.com (P.-C.Z.); qin_chunyin@163.com (C.-Y.Q.); \\ zuotianyujintian@163.com (L.F.); lingtj@ahau.edu.cn (T.-J.L.); ningjm@ahau.edu.cn (J.-M.N.) \\ 2 Institute of Fruit and Tea, Hubei Academy of Agricultural Sciences, Wuhan 430064, China; liuppitea@163.com \\ * Correspondence: zhangliang@ahau.edu.cn (L.Z.); xcwan@ahau.edu.cn (X.-C.W.) \\ + These authors contributed equally to this work.
}

Citation: Zheng, P.-C.; Qin, C.-Y.; Liu, P.-P.; Feng, L.; Ling, T.-J.; Ning, J.-M.; Zhang, L.; Wan, X.-C.

Untargeted Metabolomics Combined with Bioassay Reveals the Change in Critical Bioactive Compounds during the Processing of Qingzhuan Tea. Molecules 2021, 26, 6718. https:// doi.org/10.3390/molecules26216718

Academic Editors: Eulogio

J. Llorent-Martínez and

Gokhan Zengin

Received: 24 September 2021

Accepted: 2 November 2021

Published: 6 November 2021

Publisher's Note: MDPI stays neutral with regard to jurisdictional claims in published maps and institutional affiliations.

Copyright: (c) 2021 by the authors. Licensee MDPI, Basel, Switzerland. This article is an open access article distributed under the terms and conditions of the Creative Commons Attribution (CC BY) license (https:// creativecommons.org/licenses/by/ $4.0 /)$.

\begin{abstract}
Qingzhuan tea (QZT) is a typical Chinese dark tea that has a long-time manufacturing process. In the present study, liquid chromatography coupled with tandem mass spectrometry was used to study the chemical changes of tea samples during QZT processing. Untargeted metabolomics analysis revealed that the pile-fermentation and turnover (post-fermentation, FT) was the crucial stage in transforming the main compounds of QZT, whose contents of flavan-3-ols and flavonoids glycosides were decreased significantly. The bioactivities, including the antioxidant capacities and inhibitory effects on $\alpha$-amylase and $\alpha$-glucosidase, were also reduced after the FT process. It was suggested that although the QZT sensory properties improved following pile-fermentation and aging, the bioactivities remained restrained. Correlation analysis indicated that the main galloylated catechins and flavonoid glycosides were highly related to their antioxidant capacity and inhibitory effects on $\alpha$-amylase and $\alpha$-glucosidase.
\end{abstract}

Keywords: qingzhuan tea; metabolomics; pile-fermentation; sensory evaluation; bioactivities

\section{Introduction}

Qingzhuan tea (QZT), one of the most famous dark teas in China, has attracted significant attention due to its use of special manufacturing technology and long-time pilefermentation [1-3]. The processing steps of QZT include fixing, rolling, preliminary drying to the water content below 13\%, pile-fermentation (turnover thrice and aging 3 months), steaming, shaping, and drying [4]. Unlike other dark teas, pile-fermentation sustains QZT from several months to years [5]. After preliminary drying, raw dark teas are piled together up to 3-5 $\mathrm{m}$ in a warehouse. The chemical changes of raw dark tea are thought to occur during the long-term pile-fermentation, responsible for the unique flavor and health benefits [6].

To date, the main chemical analysis for QZT has consisted of flavan-3-ols, purine alkaloids, and some low-molecular-weight phenolic acids as the main compounds [7]. Given that the QZT raw materials consist of mature leaves and stems, the chemical composition differs from that of other typical green teas, such as Longjing tea, Maofeng tea, and some black teas $[8,9]$. Liquid chromatography-tandem mass spectrometry and metabolomics are widely used as a potent tool in tea research, despite differences in the secondary metabolites or the chemical constituents during processing [10]. For example, it was recently uncovered that the first stage of pile-fermentation has the strongest effect on the formation of $N$-ethyl-2-pyrrolidone-substituted flavan-3-ols [11].

Diabetes, a common noncommunicable chronic disease, is one of the causes of morbidity and mortality, and a global health issue [12]. Several studies have shown that 
tea possesses various health benefits, such as hypoglycemic, lipid-lowering, antioxidant, and anti-inflammatory benefits [13-15]. Among the six types of tea, dark tea has been reported to possess the ability of regulating gut microbiota, thereby mediating anti-obesity effects [16]. However, there are limited studies on the relationship between the bioactivities of compounds and the processing of dark tea. Therefore, it is necessary to study the relationship to optimize the processing technology.

Compared with Pu-erh tea and Fu brick tea [5], which belong to the same category of dark tea, reports of the chemical changes of QZT during processing are limited. In our previous study, we preliminarily studied the changes of the main compounds during pilefermentation [17] and found that the polyphenols drastically decreased at the beginning of pile-fermentation $[18,19]$. However, the changes of main compounds and trace metabolites during the whole processing steps were less studied. In the present study, we aimed to reveal the comprehensive changes of main and trace metabolites of QZT, and the relationship of the tea compounds and biological activities.

\section{Materials and Methods}

\subsection{Samples and Chemical Reagents}

To make the QZT, fresh tea leaves (FTL) were collected from the Zhaoliqiao tea factory (Chibi, China) and then fixed for $3 \mathrm{~min}$ at $280{ }^{\circ} \mathrm{C}$ (collected sample was labeled as DTL). Then, the tea leaves were rolled for $15 \mathrm{~min}$ (collected sample was labeled as RTL) by a rolling machine (6CR-55, Zhejiang, Shang Yang Co., Ltd., Quzhou, China), and finally dried in the sun (collected sample was labeled as CT). Then, CT underwent pile-fermentation after adding 30\% water, and the tea pile was turned over three times during the pile-fermentation with an interval of 7-10 days. All tea samples (after turning over) were collected and labeled as 1st FT (the first pile-fermented tea), 2nd FT (the second pile-fermented tea), 3rd FT (the third pile-fermented tea), respectively. Whereafter, the pile-fermented tea was aged for 6 months at room temperature $\left(25^{\circ} \mathrm{C}\right)$, and the aged tea leaves were collected at an interval of 3 months and labeled as 1MAT (1-month aged tea), 3MAT (3-month aged tea), 6MAT (6-month aged tea), respectively. Finally, the QZT was obtained after drying and labeled as DT (dried tea). All collected samples from the different stage of QZT processing were freeze-dried using a lyophilizer (Christ Alpha 1-4 LSC basic, Lower Saxony, Germany) and stored at $-80^{\circ} \mathrm{C}$ prior to analysis.

Gallic acid (GA, $>98 \%)$, caffeine (CAF, $>98 \%$ ), theobromine (THB, $>98 \%),(+)$-catechin $(\mathrm{C},>98 \%),(-)$ - epicatechin (EC, $>98 \%),(-)$-gallocatechin (GC, $>98 \%),(-)$-epigallocatechin (EGC, $>98 \%),(-)$ - gallocatechin gallate (GCG, >98\%), (-) —epigallocatechin gallate (EGCG, $>98 \%$ ), and (-) - epicatechin gallate (ECG, >98\%) were purchased from Yuanye Biotechnology Company (Shanghai, China). LC-MS-grade acetonitrile, methanol, and water were purchased from Thermo Fisher Scientific Co. (Fair Lawn, NJ, USA). Other reagents were of analytical grade.

\subsection{Sample Preparation}

The dried tea sample $(100 \mathrm{mg})$ was extracted with $4 \mathrm{~mL}$ of $70 \%$ methanol $(v / v)$ under ultrasonic extraction for $10 \mathrm{~min}$, and then centrifuged for $10 \mathrm{~min}$ at $6000 \mathrm{rpm}$. The tea sample was extracted again. The supernatants were combined and transferred into a 10 $\mathrm{mL}$ of the flask, and then diluted to $10 \mathrm{~mL}$ with methanol. The content was filtered via a $0.22 \mu \mathrm{m}$ nylon membrane prior to high-performance liquid chromatography (HPLC) and liquid chromatography coupled with quadrupole time of flight mass spectrometry (LC-Q-TOF-MS) analysis.

\subsection{Sensory Evaluation}

The color, odor, and taste of tea infusion and the brewed tea leaves were evaluated according to a standardized methodology for tea analysis (GB/T 23776-2018). In brief, 5.0 $\mathrm{g}$ of tea were brewed with $250 \mathrm{~mL}$ of boiled water for $5 \mathrm{~min}$. Then, the tea infusion was evaluated by panelists who were trained for 4 weeks. 


\subsection{Determination the Contents of Main Compounds by HPLC}

The HPLC system (Agilent Technologies, Palo Alto, CA, USA) which consisted of an Infinity binary pump, integrated vacuum degasser, autosampler, thermostated column compartment, and diode array detector (DAD), was used to determine the contents of tea polyphenols and purine alkaloids. The HPLC (Waters 2695, Milford, PA, USA) was coupled with a Waters Phenomenex C18 $(250 \times 4.6 \mathrm{~mm}, 5 \mu \mathrm{m})$ column, UV detector (detection wavelength set at $280 \mathrm{~nm}$ ), and a 2998 PDA detector. The temperature of the column was kept constant at $40{ }^{\circ} \mathrm{C}$. The mobile phase was composed of $2 \%(v / v)$ acetic acid (A) and acetonitrile (B). The gradient elution was set as follows: $0 \mathrm{~min}, 93.5 \% \mathrm{~A} ; 16 \mathrm{~min}, 15 \% \mathrm{~A}$; $16-25 \mathrm{~min}, 25 \% \mathrm{~A} ; 30 \mathrm{~min}, 93.5 \% \mathrm{~A}$; and $40 \mathrm{~min}, 93.5 \% \mathrm{~A}$. The injection volume was $10 \mu \mathrm{L}$ and the flow rate was $1.0 \mathrm{~mL} / \mathrm{min}$. The analytical method was based on our previously published method [20].

\subsection{Untargeted Metabolomics Analysis}

The metabolites of QZT samples were analyzed using an Agilent 1290 LC system (Agilent Technologies, Palo Alto, CA, USA) coupled to a time-of-flight mass spectrometer (Agilent Technologies, Palo Alto, CA, USA). The separation of the chemical compounds was the same as our established method [13]. Samples analyses were performed in triplicate. The mass spectrometer was operated in the negative ionization mode at a resolving power of 40,000 over a full scan range of $m / z$ 100-1200 with the following settings: sheath gas temperature, $350{ }^{\circ} \mathrm{C}$; nebulizer, $35 \mathrm{psi}$; gas flow, $8 \mathrm{~L} / \mathrm{min}$; gas temperature, $320^{\circ} \mathrm{C}$; sheath gas flow, $11 \mathrm{~L} / \mathrm{min}$.

SMICA-P 13.0 software (Umetrics, Umeå, Sweden) was used for multivariate analysis based the LC-MS data. Data within a 95\% confidence interval were accepted.

\subsection{Inhibitory Effects of QZT Samples on $\alpha$-Amylase and $\alpha$-Glucosidase}

The QZT samples from different processing stages were prepared similarly to the method described in Section 2.2. Then, the tea infusions were diluted to various concentrations for the determination of their inhibition effects on $\alpha$-amylase and $\alpha$-glucosidase. The in vitro inhibition of $\alpha$-amylase and $\alpha$-glucosidase of each tea sample was assessed using a previously described methodology, and results were expressed as the inhibition rate [20].

\subsection{In Vitro Antioxidant Assay}

The in vitro antioxidant activity of each tea sample was evaluated by the following three tests. The ferric reducing ability of plasma (FRAP) was determined according to the method proposed by Benzie and Strain (1997), and the results were expressed as the FRAP value [21]. The antioxidant activity in relation to the 2, 2-diphenyl-1-picrylhydrazyl (DPPH) radical was determined by the method proposed by Brand-Williams et al. (1995), and the results were expressed as the DPPH scavenging rate and $\mathrm{IC}_{50}$ value [22]. For the ABTS assay, the antioxidant activity of each sample was determined according to the published method [23].

\subsection{Statistical Analysis}

All samples were analyzed in triplicate, and the results were expressed as mean \pm $\mathrm{SD}(\mathrm{n}=3)$. One-way ANOVA followed by Tukey's test was used to separate the means. $p$-values below $5 \%$ were considered significant.

\section{Results and Discussion}

\subsection{Sensory Evaluation}

As shown in Figure 1, with the depth of QZT processing, the color of tea infusion and tea leaves gradually turned red and darker. Furthermore, the astringent scores of the processed tea samples showed a significant variation during processing. This suggested that tea leaves underwent dramatic changes in chemical compositions during the QZT processing. Initially, the astringent score was gradually increased along with the processing 
steps, but after pile-fermentation and aging of crude tea, the astringent score was dramatically decreased. Hence, the flavor of QZT may be mainly formed at the pile-fermentation and aging steps, especially the third FT and 1MAT. The enzymatic oxidation of tea polyphenols has been inhibited or terminated due to the polyphenol oxidase was deactivated during the fixation of fresh tea leaves, whereas the oxidation of tea polyphenols and other processing-derived products might play an important role in the color and taste formation of QZT products [24].

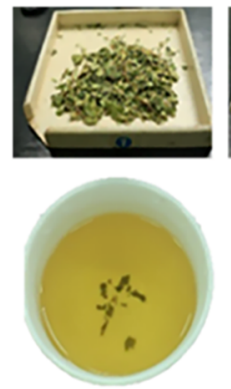

FTL

1
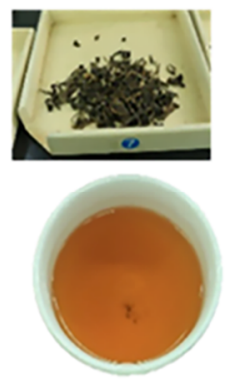

3rd FT
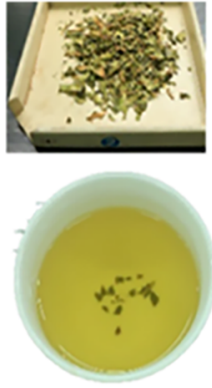

DTL
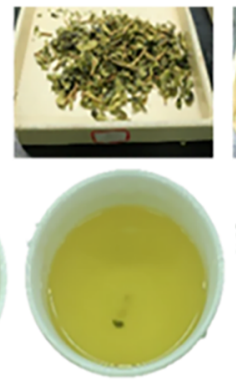

RTL
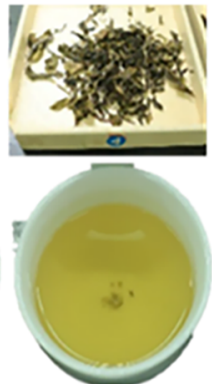

CT

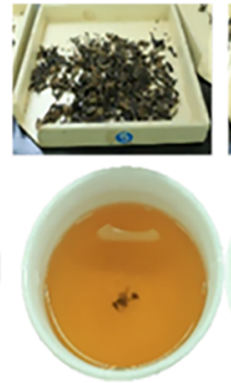

1st FT

Astringent Score

3

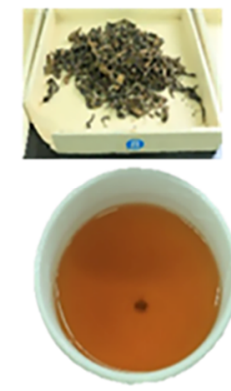

3MAT
4

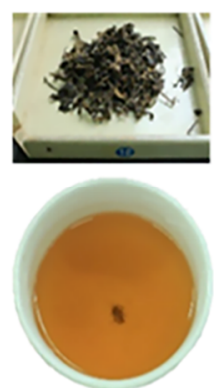

6MAT

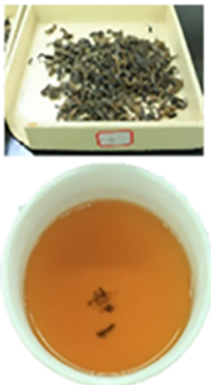

2nd FT

1

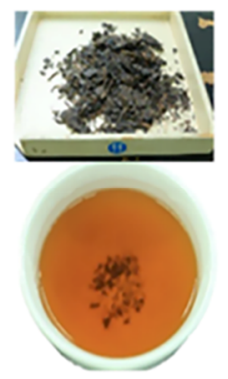

DT

3

2

Astringent Score

2

1

Figure 1. Sensory evaluation on the color and taste (mainly astringency) of QZT samples from the different processing stages. The first line above the abbreviations of tea samples showed the color of tea leaves, and the second line showed the color of tea infusions. The numbers under the abbreviations of tea samples represent their corresponding astringent scores.

\subsection{Contents of Main Compounds in QZT Samples}

The contents of the main compounds in the QZT samples from different processing steps are presented in Table 1 . The aqueous extract is an important factor in evaluating the quality of tea samples [25]. With the processing of QZT, the content of aqueous extracts (\%) did not show significant changes, but it was statistically declined. Furthermore, the contents of total polyphenols, amino acids, flavonoids, and soluble sugars were continuously decreased along with processing. These results are consistent with most of the similar reports for Chinese dark teas, such as Pu-erh tea and Fuzhuan tea [25-27]. 
Table 1. The contents of aqueous extracts, total polyphenols, total amino acids, total flavonoids, soluble sugar, gallic acid, caffeine, and theobromine of different processed tea samples $(\% w t / w t)$.

\begin{tabular}{|c|c|c|c|c|c|c|c|c|}
\hline Samples & $\begin{array}{l}\text { Aqueous } \\
\text { Extracts }\end{array}$ & $\begin{array}{c}\text { Total } \\
\text { Polyphenols }\end{array}$ & $\begin{array}{l}\text { Total Amino } \\
\text { Acids }\end{array}$ & $\begin{array}{c}\text { Total } \\
\text { Flavonoids }\end{array}$ & $\begin{array}{l}\text { Total Soluble } \\
\text { Sugars }\end{array}$ & Gallic Acid & Caffeine & Theobromine \\
\hline FTL & $37.71 \pm 2.21^{\mathrm{a}}$ & $18.18 \pm 1.06^{\mathrm{a}}$ & $1.37 \pm 0.08^{a}$ & $2.24 \pm 0.03^{a}$ & $5.92 \pm 0.62^{a}$ & $0.08 \pm 0.00^{c}$ & $2.80 \pm 0.16^{\mathrm{a}, \mathrm{b}}$ & $0.08 \pm 0.00^{\mathrm{d}}$ \\
\hline DTL & $35.57 \pm 0.51^{\mathrm{a}, \mathrm{b}}$ & $16.33 \pm 1.11^{\mathrm{a}, \mathrm{b}}$ & $1.13 \pm 0.04^{\mathrm{a}, \mathrm{b}}$ & $2.07 \pm 0.02^{b}$ & $5.49 \pm 1.18^{a}$ & $0.09 \pm 0.00^{c}$ & $2.57 \pm 0.09^{c, d}$ & $0.07 \pm 0.00^{\mathrm{e}}$ \\
\hline RTL & $36.31 \pm 0.63^{a, b}$ & $15.99 \pm 0.85^{b}$ & $0.95 \pm 0.04^{b}$ & $2.09 \pm 0.02^{b}$ & $5.69 \pm 0.49^{a}$ & $0.07 \pm 0.01^{\mathrm{c}}$ & $2.50 \pm 0.11^{\mathrm{c}, \mathrm{d}}$ & $0.07 \pm 0.00^{f}$ \\
\hline $\mathrm{CT}$ & $35.47 \pm 0.52^{\mathrm{a}, \mathrm{b}}$ & $15.61 \pm 0.49^{b}$ & $0.83 \pm 0.25^{b, c}$ & $1.95 \pm 0.09^{b}$ & $4.93 \pm 0.05^{\mathrm{a}, \mathrm{b}}$ & $0.15 \pm 0.01^{b c}$ & $2.92 \pm 0.15^{a}$ & $0.09 \pm 0.00^{b, c}$ \\
\hline 1st FT & $34.69 \pm 0.15^{b}$ & $12.87 \pm 0.34^{\mathrm{c}}$ & $0.76 \pm 0.12^{b, c}$ & $1.78 \pm 0.11^{c}$ & $6.05 \pm 0.39^{a, b}$ & $0.07 \pm 0.00^{c}$ & $2.59 \pm 0.09^{c}$ & $0.09 \pm 0.00^{c}$ \\
\hline 2nd FT & $35.14 \pm 0.29^{b, c}$ & $9.77 \pm 0.97^{\mathrm{d}}$ & $0.69 \pm 0.06^{b, c}$ & $1.63 \pm 0.05^{\mathrm{d}}$ & $4.79 \pm 0.46^{\mathrm{a}, \mathrm{b}}$ & $0.08 \pm 0.01^{c}$ & $2.70 \pm 0.13^{b}$ & $0.10 \pm 0.00^{b}$ \\
\hline 3rd FT & $34.70 \pm 0.33^{b, c}$ & $7.08 \pm 0.23 \mathrm{e}$ & $0.57 \pm 0.04^{c}$ & $1.55 \pm 0.03^{\mathrm{d}, \mathrm{e}}$ & $3.99 \pm 0.52^{a, b}$ & $0.46 \pm 0.04^{\mathrm{a}}$ & $2.81 \pm 0.10^{b}$ & $0.08 \pm 0.00^{\mathrm{e}}$ \\
\hline 1MAT & $34.16 \pm 0.38^{b, c}$ & $5.58 \pm 0.24^{\mathrm{e}, \mathrm{f}}$ & $0.53 \pm 0.02^{c, d}$ & $1.51 \pm 0.02^{\mathrm{d}, \mathrm{e}}$ & $3.26 \pm 0.42^{b}$ & $0.43 \pm 0.04^{\mathrm{a}}$ & $2.65 \pm 0.15^{b, c}$ & $0.09 \pm 0.00^{\mathrm{c}, \mathrm{d}}$ \\
\hline 3MAT & $34.03 \pm 0.52^{b, c}$ & $5.14 \pm 0.11^{\mathrm{f}}$ & $0.51 \pm 0.03^{c, d}$ & $1.47 \pm 0.02 \mathrm{e}$ & $3.32 \pm 0.53^{b}$ & $0.43 \pm 0.02^{\mathrm{a}}$ & $2.54 \pm 0.14^{c}$ & $0.06 \pm 0.00^{f}$ \\
\hline $6 \mathrm{MAT}$ & $34.19 \pm 0.43^{b, c}$ & $4.52 \pm 0.31^{\mathrm{f}}$ & $0.47 \pm 0.01^{\mathrm{d}}$ & $1.43 \pm 0.02^{\mathrm{e}}$ & $3.33 \pm 0.30^{b}$ & $0.37 \pm 0.01^{\mathrm{a}}$ & $2.38 \pm 0.13^{d}$ & $0.08 \pm 0.00^{\mathrm{e}}$ \\
\hline DT & $32.72 \pm 0.91^{\mathrm{c}}$ & $4.16 \pm 0.17^{\mathrm{f}}$ & $0.43 \pm 0.01^{\mathrm{d}}$ & $1.44 \pm 0.03^{\mathrm{e}}$ & $3.25 \pm 0.21^{b}$ & $0.25 \pm 0.02^{b}$ & $2.47 \pm 0.13^{c, d}$ & $0.11 \pm 0.01^{\mathrm{a}}$ \\
\hline
\end{tabular}

Note: The abbreviations for samples are FTL: fresh tea leaves; DTL: deactivated tea leaves; RTL: rolled tea leaves; CT: crude tea; 1st FT: the first pile-fermented tea; 2nd FT: the second pile-fermented tea; 3rd FT: the third pile-fermented tea; 1MAT: 1-month aged tea; 3MAT: 3-month aged tea; 6MAT: 6-month aged tea; and DT: dried tea. Different letters in the same column indicated that the contents of chemical compounds had significant differences $(p<0.05)$.

Gallic acid was gradually accumulated during the QZT processing. Specially, after the third pile-fermentation, its content (\%) attained the highest value at $0.46 \%$ (Table 1$)$. However, similar to the other reports of Chinese dark tea [28], the content of caffeine was relatively stable. In the beginning, the fresh tea leaves contained $2.80 \%$ of caffeine, but after whole processing, the caffeine content remained at $2.47 \%$.

\subsection{Contents of Catechins during Processing}

As shown in Table 2, the content of each catechin compound was determined. EGCG was the main polyphenol in the fresh tea leaves, which was continuously decreased with processing. The crude tea leaves also had a higher content of EGCG. However, after three times of pile-fermentation, its content was significantly decreased. Other galloylated catechins showed a similar variation, and the contents of non-galloylated catechins, including GC, EGC, C, and EC, were also decreased. These results suggest the hydrolysis of galloylated catechins may not produce non-galloylated catechins as the final product. The non-galloylated catechins were also decreased, and then the total catechins were greatly reduced. Subsequently, the astringent perception was correspondingly changed.

Table 2. The contents of catechins in QZT samples from different processing stages ( $\%$ wt/wt).

\begin{tabular}{|c|c|c|c|c|c|c|c|c|c|c|}
\hline Samples & GC & EGC & C & EGCG & EC & GCG & ECG & CG & $\begin{array}{c}\text { Total } \\
\text { Catechins }\end{array}$ & $\begin{array}{l}\text { Galloylated/ } \\
\text { Non- } \\
\text { Galloylated } \\
\text { Catechins }\end{array}$ \\
\hline FTL & $0.98 \underset{\mathrm{a}}{ \pm 0} 0.02$ & $1.36 \underset{\mathrm{a}}{ \pm 0.04}$ & $0.22 \pm 0.01$ & $3.28 \underset{\mathrm{a}}{ \pm 0.09}$ & $0.62 \underset{\mathrm{a}, \mathrm{b}}{ \pm} 0.03$ & $0.84 \underset{\mathrm{b}}{ \pm} 0.04$ & $0.94 \underset{\mathrm{a}}{ \pm 0.04}$ & $0.17 \underset{\mathrm{b}, \mathrm{c}}{ \pm} 0.01$ & $8.41 \pm \underset{\mathrm{a}}{ \pm} 0.17$ & $1.64 \underset{\mathrm{c}, \mathrm{d}}{ \pm} 0.02$ \\
\hline DTL & $0.91 \pm 0.01$ & $1.18 \frac{ \pm}{\mathrm{b}} 0.05$ & $0.25 \underset{\mathrm{a}}{ \pm} 0.00$ & $3.03 \underset{\mathrm{a}, \mathrm{b}}{ \pm} 0.10$ & $0.54 \underset{b, c}{ \pm} 0.01$ & $1.01 \pm \underset{\mathrm{a}}{ \pm} 0.07$ & $0.79 \frac{ \pm}{\mathrm{b}} 0.02$ & $0.20 \underset{\mathrm{a}, \mathrm{b}}{ \pm} 0.01$ & $7.91 \underset{\mathrm{a}, \mathrm{b}}{ \pm} 0.19$ & $1.74 \underset{\mathrm{b}}{ \pm} 0.02$ \\
\hline RTL & $0.64 \underset{c}{ \pm} 0.03$ & $1.37 \underset{\mathrm{a}}{ \pm} 0.04$ & $0.19 \underset{c}{ \pm} 0.01$ & $3.08 \underset{\mathrm{a}, \mathrm{b}}{ \pm} 0.08$ & $0.50 \underset{c}{ \pm} 0.01$ & $0.81 \underset{\mathrm{b}}{ \pm} 0.04$ & $0.83 \frac{ \pm}{\mathrm{b}} 0.03$ & $0.16 \underset{\mathrm{b}, \mathrm{c}}{ \pm} 0.01$ & $7.58 \underset{\mathrm{b}}{ \pm} 0.14$ & $1.80 \underset{\mathrm{a}}{ \pm} 0.03$ \\
\hline $\mathrm{CT}$ & $0.65 \underset{c}{ \pm} 0.01$ & $1.22 \frac{ \pm}{\mathrm{b}} 0.03$ & $0.18 \underset{c}{ \pm} 0.01$ & $2.85 \frac{ \pm}{\mathrm{b}} 0.08$ & $0.67 \underset{\mathrm{a}}{ \pm} 0.03$ & $0.58 \underset{c}{ \pm} 0.03$ & $0.82 \frac{ \pm}{\mathrm{b}} 0.03$ & $0.12 \underset{c}{ \pm} 0.01$ & $7.09 \underset{b, c}{ \pm} 0.15$ & $1.60 \pm \frac{\mathrm{d}}{ \pm} 0.03$ \\
\hline 1st FT & $0.56 \underset{\mathrm{d}}{ \pm 0.02}$ & $1.00 \underset{c}{ \pm} 0.06$ & $0.24 \underset{\mathrm{a}, \mathrm{b}}{ \pm} 0.01$ & $2.70 \underset{\mathrm{bc}}{ \pm} 0.12$ & $0.57 \underset{\mathrm{b}}{ \pm} 0.03$ & $0.42 \underset{\mathrm{d}}{ \pm} 0.03$ & $0.75 \underset{\mathrm{b}}{ \pm} 0.02$ & $\underset{\mathrm{a}}{0.23 \pm 0.02}$ & $6.47 \underset{c}{ \pm} 0.18$ & $1.73 \underset{\mathrm{b}}{ \pm} 0.02$ \\
\hline 2nd FT & $0.60 \underset{c, d}{ \pm} 0.01$ & $1.00 \underset{c}{ \pm} 0.02$ & $0.18 \underset{c}{ \pm} 0.01$ & $2.20 \underset{c}{ \pm} 0.06$ & $0.43 \underset{\mathrm{d}}{ \pm} 0.03$ & $0.62 \underset{c}{ \pm} 0.03$ & $0.60 \underset{c}{ \pm 0} 0.02$ & $0.11 \underset{c, d}{ \pm} 0.01$ & $5.74 \underset{\mathrm{c}, \mathrm{d}}{ \pm} 0.12$ & $1.60 \underset{\mathrm{d}, \mathrm{e}}{ \pm} 0.04$ \\
\hline 3rd FT & $0.40 \pm \frac{\mathrm{e}}{\mathrm{e}} 0.02$ & $0.85 \underset{\mathrm{d}}{ \pm} 0.04$ & $0.14 \underset{\mathrm{d}}{ \pm} 0.01$ & $1.62 \pm 0.08$ & $0.29 \underset{\mathrm{e}, \mathrm{f}}{ \pm} 0.03$ & $0.48 \underset{\mathrm{d}}{ \pm} 0.04$ & $0.50 \pm 0.01$ & $0.17 \underset{\mathrm{b}}{ \pm} 0.02$ & $4.45 \underset{\mathrm{d}}{ \pm} 0.15$ & $1.66 \underset{c}{ \pm} 0.01$ \\
\hline 1MAT & $0.38 \underset{\mathrm{e}}{ \pm 0.01}$ & $0.63 \underset{\mathrm{e}}{ \pm 0.02}$ & $0.11 \underset{\mathrm{f}}{ \pm} 0.00$ & $1.06 \underset{\mathrm{e}}{ \pm 0.03}$ & $0.23 \underset{\mathrm{f}}{ \pm} 0.02$ & $0.27 \underset{\mathrm{e}}{ \pm 0.01}$ & $0.36 \underset{\mathrm{e}, \mathrm{f}}{ \pm} 0.02$ & $0.09 \underset{\mathrm{c}, \mathrm{d}}{ \pm} 0.01$ & $3.13 \pm \underset{\mathrm{e}}{ \pm 0.08}$ & $1.32 \underset{\mathrm{g}}{ \pm} 0.02$ \\
\hline 3MAT & $0.30 \underset{\mathrm{f}}{ \pm} 0.01$ & $0.46 \underset{\mathrm{f}}{ \pm} 0.01$ & $0.11 \underset{\mathrm{e}, \mathrm{f}}{ \pm} 0.01$ & $0.98 \underset{\mathrm{e}}{ \pm 0.03}$ & $0.32 \underset{\mathrm{e}}{ \pm} 0.01$ & $0.28 \pm \underset{\mathrm{e}}{0} 0.01$ & $0.42 \pm \frac{\mathrm{e}}{0.02}$ & $0.08 \underset{\mathrm{d}}{ \pm} 0.02$ & $2.95 \pm \underset{\mathrm{e}}{ \pm 0.07}$ & $1.48 \underset{\mathrm{f}}{ \pm} 0.03$ \\
\hline $6 \mathrm{MAT}$ & $0.14 \underset{\mathrm{g}}{ \pm} 0.01$ & $0.27 \pm 0.01$ & $0.13 \underset{\mathrm{d}, \mathrm{e}}{ \pm} 0.01$ & $0.67 \underset{\mathrm{f}}{ \pm} 0.02$ & $0.29 \underset{\mathrm{e}, \mathrm{f}}{ \pm} 0.02$ & $0.23 \pm \underset{\mathrm{e}}{0.01}$ & $0.32 \underset{\mathrm{f}}{ \pm} 0.01$ & $0.09 \underset{\mathrm{c}, \mathrm{d}}{ \pm} 0.02$ & $2.14 \underset{\mathrm{f}}{ \pm} 0.05$ & $1.57 \underset{\mathrm{d}, \mathrm{e}}{ \pm} 0.04$ \\
\hline DT & $0.15 \underset{\mathrm{g}}{ \pm} 0.03$ & $0.23 \underset{\mathrm{g}}{ \pm} 0.01$ & $0.09 \underset{\mathrm{f}}{ \pm} 0.00$ & $0.20 \underset{\mathrm{g}}{ \pm} 0.02$ & $0.24 \underset{\mathrm{f}}{ \pm} 0.01$ & $0.14 \underset{\mathrm{f}}{ \pm} 0.01$ & $0.35 \underset{\mathrm{e}, \mathrm{f}}{ \pm} 0.02$ & $0.08 \underset{c, d}{ \pm} 0.01$ & $1.48 \underset{\mathrm{f}}{ \pm} 0.04$ & $1.09 \underset{\mathrm{h}}{ \pm} 0.08$ \\
\hline
\end{tabular}

Note: The abbreviations for samples are FTL: fresh tea leaves; DTL: deactivated tea leaves; RTL: rolled tea leaves; CT: crude tea; 1st FT: the first pile-fermented tea; 2nd FT: the second pile-fermented tea; 3rd FT: the third pile-fermented tea; 1MAT: 1-month aged tea; 3MAT: 3-month aged tea; 6MAT: 6-month aged tea; and DT: dried tea. Different letters in the same column indicated that the contents of chemical compounds had significant differences $(p<0.05)$. 


\subsection{Multivariate Analysis of LC-MS Based Metabolomics Data}

All of MS data files were uploaded to the MS-DIAL (version 2.74) for data processing, including the retention time $(\mathrm{min}), \mathrm{m} / \mathrm{z}$ value, and intensity alignment. The processed dataset was analyzed by SMICA-P 13.0 multivariate statistical software. The unsupervised principal composition analysis (PCA), supervised partial least squares discriminant analysis (PLS-DA), and orthonormal partial least squares discriminant analysis (OPLS-DA) discriminated all tested tea samples into two categories (Figure 2). One cluster contained FTL, DTL, RTL, and CT, while the other tea samples (1st FT, 2nd FT, 3rd FT, 1MAT, 3MAT, $6 \mathrm{MAT}$, and DT) were grouped in the same cluster. The hierarchical cluster analysis (HCA) also distinguished all samples in the same way. These results indicated that the first four processing steps, including FTL, DTL, RTL, and CT, which were also the main steps for processing green tea, had similar chemical constituents. Once the tea sample underwent pile-fermentation, the chemical constitutes changed.
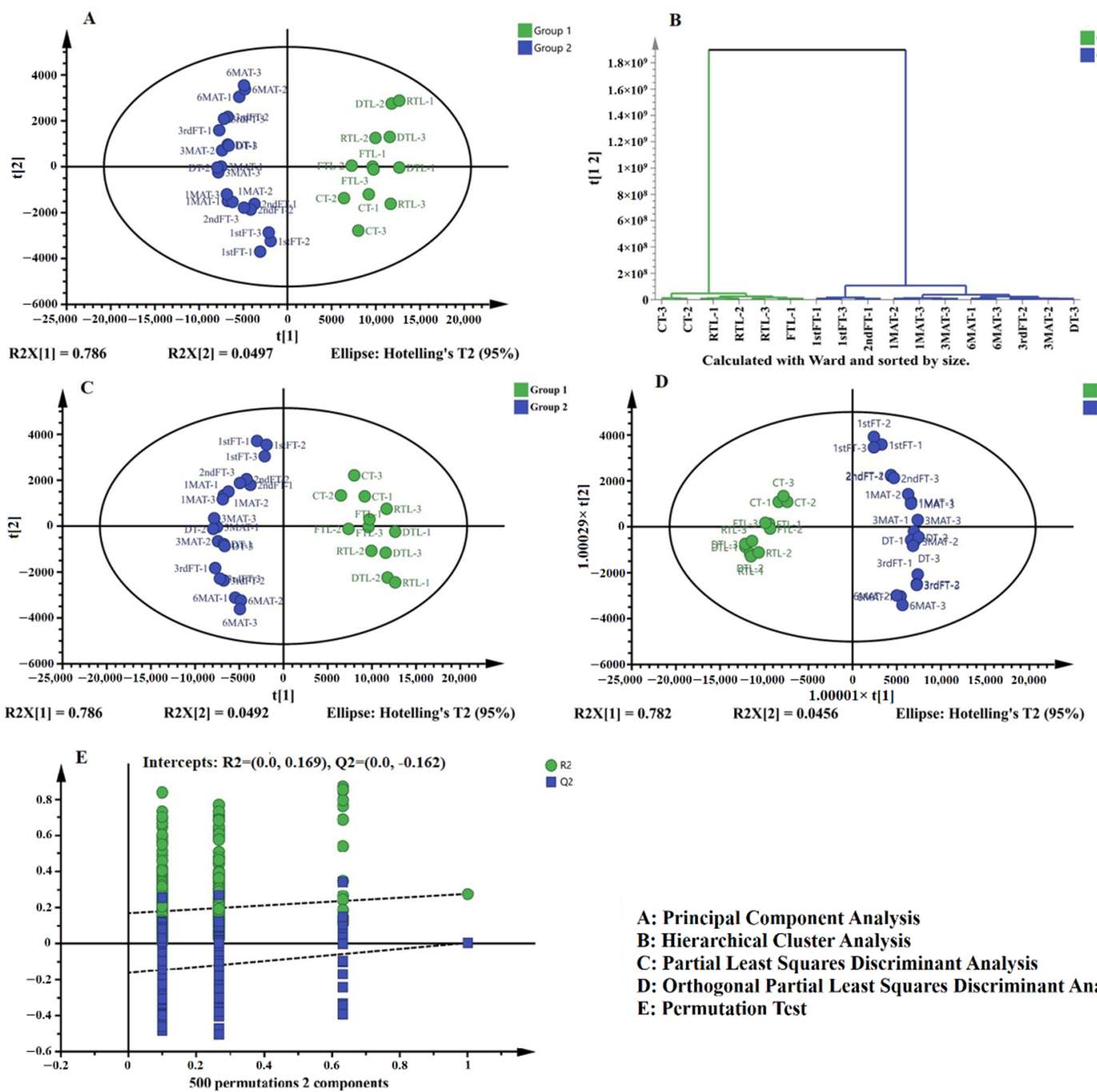

\author{
A: Principal Component Analysis \\ B: Hierarchical Cluster Analysis \\ C: Partial Least Squares Discriminant Analysis \\ D: Orthogonal Partial Least Squares Discriminant Analysis \\ E: Permutation Test
}

Figure 2. Multivariate analysis based on the LC-Q-TOF-MS data of QZT samples from different processing stages.

To further explore the marker compounds responsible for the variation between these two categories of tea samples, the scatterplots and S-plot were profiled, as shown in Figure 3. All compounds having VIP >1 were considered as important compounds (marker compounds) responsible for the classification of QZT samples. In total, 41 marker compounds were tentatively identified in Table 3 by referencing the chemical standards and mass fragments. These compounds mainly belonged to the polyphenols, which were susceptible to 
the pile-fermentation process. Among these compounds, galloylated catechins primarily contributed to the changes of chemical constitutes, such as EGCG, EGC, C, and ECG. Furthermore, some astringent compounds were also identified as marker compounds, which suggested the content decreasing of these compounds may attribute to the astringent score decreasing of tea samples, for instance, flavonoid glycosides and procyanidins.
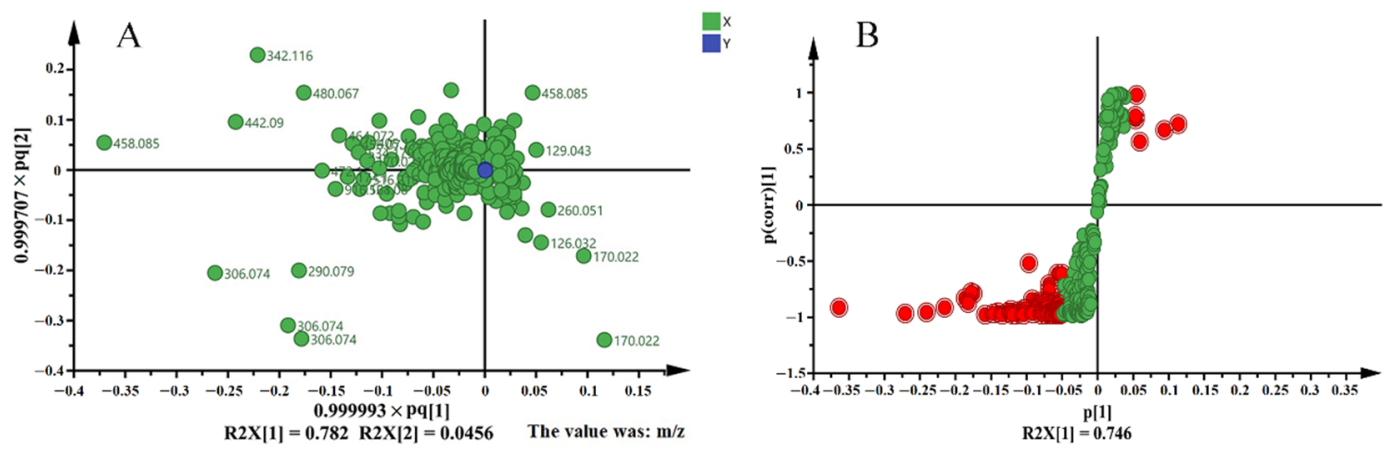

Figure 3. Metabolomics analysis of QZT samples from different processing stages. (A) Scatterplots based on OPLS-DA analysis between the two categories of QZT samples. (B) S-plots based on OPLS-DA analysis between the two categories of QZT samples.

Table 3. The critical compounds responsible for the classification of QZT samples from different processing stages.

\begin{tabular}{|c|c|c|c|c|}
\hline No. & $t_{R}(\min )$ & MW & VIP Value & Identification \\
\hline 1 & 6.282 & 458.085 & 5.67 & $(-)$-Epigallocatechin gallate \\
\hline 2 & 3.141 & 306.074 & 4.19 & (-)-Epigallocatechin \\
\hline 3 & 4.659 & 290.079 & 3.82 & $(-)$-Catechin \\
\hline 4 & 0.484 & 342.116 & 3.68 & Coniferin \\
\hline 5 & 7.780 & 442.090 & 3.48 & $(-)$-Epicatechin gallate \\
\hline 6 & 0.934 & 170.022 & 3.24 & Gallic acid \\
\hline 7 & 6.283 & 480.067 & 3.07 & Myricetin-3-O-galatoside \\
\hline 8 & 7.638 & 472.100 & 2.20 & Methoxy-epigallocatechin gallate \\
\hline 9 & 7.780 & 464.072 & 2.05 & Quercetin-3-O-glucopyranoside \\
\hline 10 & 6.282 & 916.168 & 2.04 & Assamicain A/B/C \\
\hline 11 & 6.283 & 456.070 & 1.87 & Methoxy-epicatechin gallate \\
\hline 12 & 0.501 & 174.100 & 1.85 & L-theanine \\
\hline 13 & 0.497 & 534.179 & 1.75 & Unknown \\
\hline 14 & 6.282 & 521.080 & 1.73 & Unknown \\
\hline 15 & 0.483 & 388.122 & 1.66 & Dihydrovomifoliol-O-glucopyranoside \\
\hline 16 & 0.493 & 516.216 & 1.64 & Di-caffeoylquinic acid \\
\hline 17 & 0.484 & 405.112 & 1.64 & Unknown \\
\hline 18 & 3.171 & 307.077 & 1.63 & Unknown \\
\hline 19 & 0.551 & 406.109 & 1.53 & Unknown \\
\hline 20 & 3.027 & 304.058 & 1.48 & Taxifolin \\
\hline 21 & 7.160 & 458.085 & 1.45 & $(-)-$ Gallocatechin gallate \\
\hline 22 & 7.637 & 494.083 & 1.43 & Unknown \\
\hline 23 & 3.143 & 374.061 & 1.43 & Unknown \\
\hline 24 & 1.051 & 344.074 & 1.42 & Theogallin \\
\hline 25 & 0.934 & 126.032 & 1.41 & Unknown \\
\hline 26 & 4.662 & 358.066 & 1.41 & Junipetrioloside A \\
\hline 27 & 6.280 & 548.054 & 1.38 & Unknown \\
\hline 28 & 8.664 & 456.106 & 1.28 & $(-)$-Epicatechin gallate-3"--O-Me \\
\hline 32 & 0.481 & 182.079 & 1.21 & Unknown \\
\hline 34 & 5.104 & 578.142 & 1.11 & Procyanidin B2 \\
\hline 35 & 6.284 & 938.15 & 1.09 & Unknown \\
\hline 37 & 6.278 & 454.054 & 1.06 & Unknown \\
\hline 38 & 1.044 & 366.056 & 1.05 & Unknown \\
\hline 39 & 6.281 & 478.051 & 1.03 & 7-methoxy-kaempferol-glucopyranoside \\
\hline 41 & 7.790 & 772.206 & 1.01 & $\begin{array}{l}\text { Quercetin 3-O-glucopyranosyl-O- } \\
\text { glucopyranosyl-O-rhamnoside }\end{array}$ \\
\hline
\end{tabular}

Note: The abbreviations in the table are $t_{R}$ : retention time; MW: molecular weight; VIP value: the value of the variable influence on projection. The critical compounds are listed in order of VIP value from greatest to least. 
Based on the above results, it was obvious that first pile-fermentation was the critical processing step for the transformation of chemical constituents in QZT. Our previous studies only concerned the chemical changes of pile-fermentation, while the first and second pile-fermentation were also recognized as important processing steps [17]. Thus, in the present study, we comprehensively studied the chemical compounds in QZT samples from various processing stages (in total, 11 processed tea samples were collected and analyzed) and found that significantly more marker compounds were affected by QZT processing. To correlate the relationship between chemistry and biological activities, all processed samples were studied with respect to their antioxidant capacities and the inhibitory effects on $\alpha$-amylase and $\alpha$-glucosidase. The contribution of each compound to biological activity was also calculated by the correlation coefficient.

\subsection{Inhibitory Effects on $\alpha$-Amylase and $\alpha$-Glucosidase}

In Figure 4, an apparent regulation could be observed, as the RTL and CT samples showed the best inhibition effects on both $\alpha$-amylase and $\alpha$-glucosidase. The two samples belonged to the unfermented tea samples, because since the first FT, the crude tea started pilefermentation for a different time. This suggested that the original compounds of QZT before pile-fermentation showed stronger inhibition effects on $\alpha$-amylase and $\alpha$-glucosidase, with lower $\mathrm{IC}_{50}$ values at $29.47-63.48 \mathrm{mg} / \mathrm{mL}$ ( $\alpha$-amylase) and 52.91-69.57 $\mu \mathrm{g} / \mathrm{mL}(\alpha$-glucosidase). Correspondingly, in Table 2, the contents of the main catechins were significantly decreased after the first fermentation and turnover (1st FT). The non-targeted metabolomics results also confirmed that the first four samples were classified into the same category.
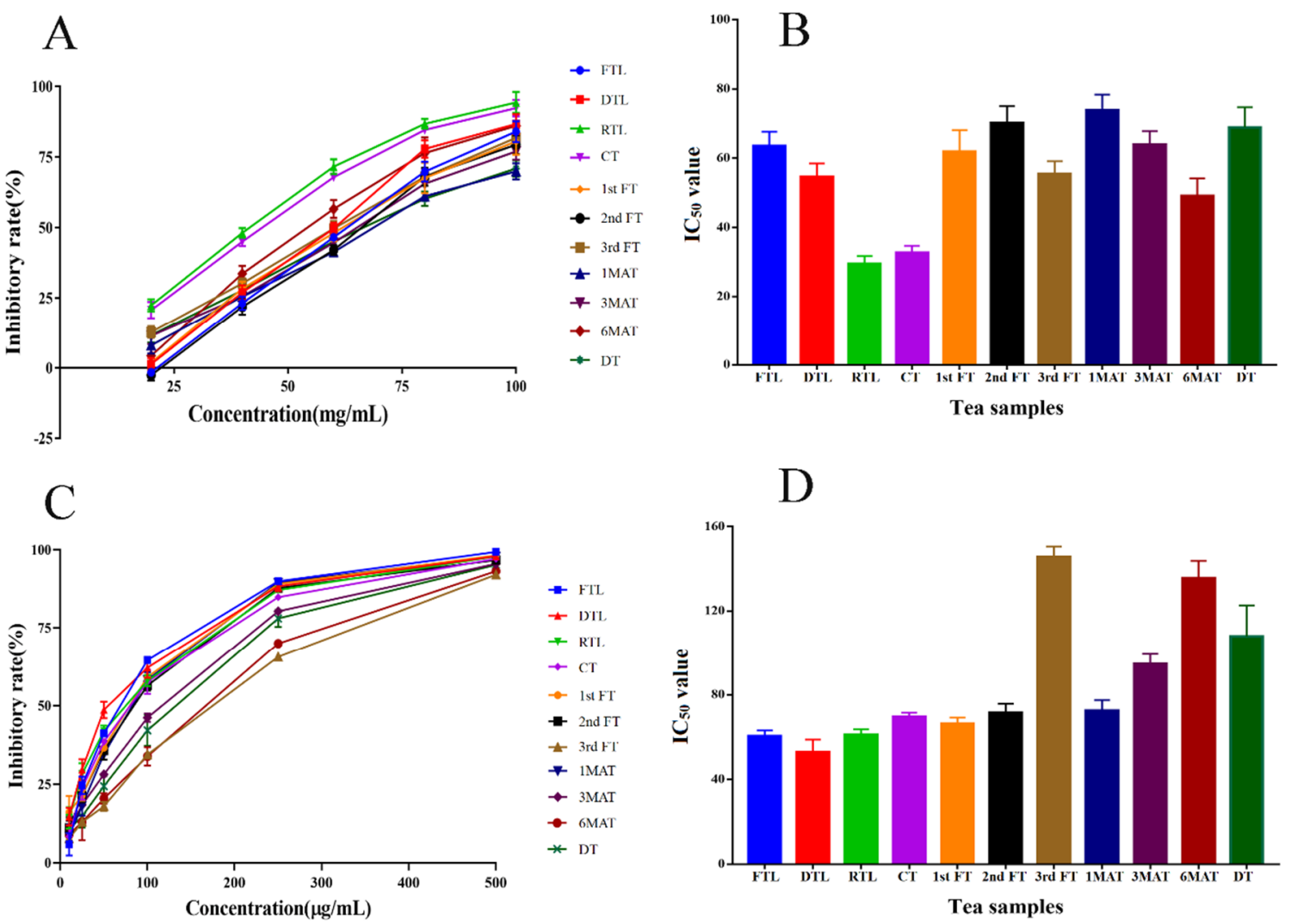

Figure 4. The inhibitory effects of different QZT samples on $\alpha$-amylase and $\alpha$-glucosidase. (A) Inhibitory rates of different QZT samples with various concentrations on $\alpha$-amylase. (B) $\mathrm{IC}_{50}$ values of different QZT samples on $\alpha$-amylase: FTL: $63.48 \mathrm{mg} / \mathrm{mL}$; DTL: $54.59 \mathrm{mg} / \mathrm{mL}$; RTL: $29.47 \mathrm{mg} / \mathrm{mL}$; CT: 32.59 mg/mL; 1st FT: $61.93 \mathrm{mg} / \mathrm{mL}$; 2nd FT: $70.16 \mathrm{mg} / \mathrm{mL}$; 3rd FT: $55.42 \mathrm{mg} / \mathrm{mL}$; 1MAT: $73.86 \mathrm{mg} / \mathrm{mL}$; 3MAT: $63.98 \mathrm{mg} / \mathrm{mL}$; 6MAT: $49.07 \mathrm{mg} / \mathrm{mL}$; DT: $68.88 \mathrm{mg} / \mathrm{mL}$. (C) Inhibitory rates of different QZT samples with various concentrations on $\alpha$-glucosidase. (D) $\mathrm{IC}_{50}$ values of different QZT samples on $\alpha$-glucosidase: FTL: $60.54 \mu \mathrm{g} / \mathrm{mL}$; DTL: $52.91 \mu \mathrm{g} / \mathrm{mL}$; RTL: $61.01 \mu \mathrm{g} / \mathrm{mL}$; CT: $69.57 \mu \mathrm{g} / \mathrm{mL}$; 1st FT: $66.26 \mu \mathrm{g} / \mathrm{mL} ; 2 \mathrm{nd} \mathrm{FT:}$ $71.60 \mu \mathrm{g} / \mathrm{mL}$; 3rd FT: $145.60 \mu \mathrm{g} / \mathrm{mL} ; 1 \mathrm{MAT}: 72.54 \mu \mathrm{g} / \mathrm{mL}$; 3MAT: $94.72 \mu \mathrm{g} / \mathrm{mL} ; 6 \mathrm{MAT}: 135.57 \mu \mathrm{g} / \mathrm{mL}$; DT: $107.94 \mu \mathrm{g} / \mathrm{mL}$. 


\subsection{Antioxidant Activities}

Figure 5 demonstrates the effect of different tea samples on antioxidant activity and the radical scavenging ability. We observed similar results to the inhibition effects on $\alpha$-amylase and $\alpha$-glucosidase. The samples before pile-fermentation and turnover (before the 1st FT) had stronger antioxidant activities compared with the pile-fermented and aged samples. This suggested that a lower content of total phenolic compounds in tea samples were strongly correlated to their antioxidant activity.
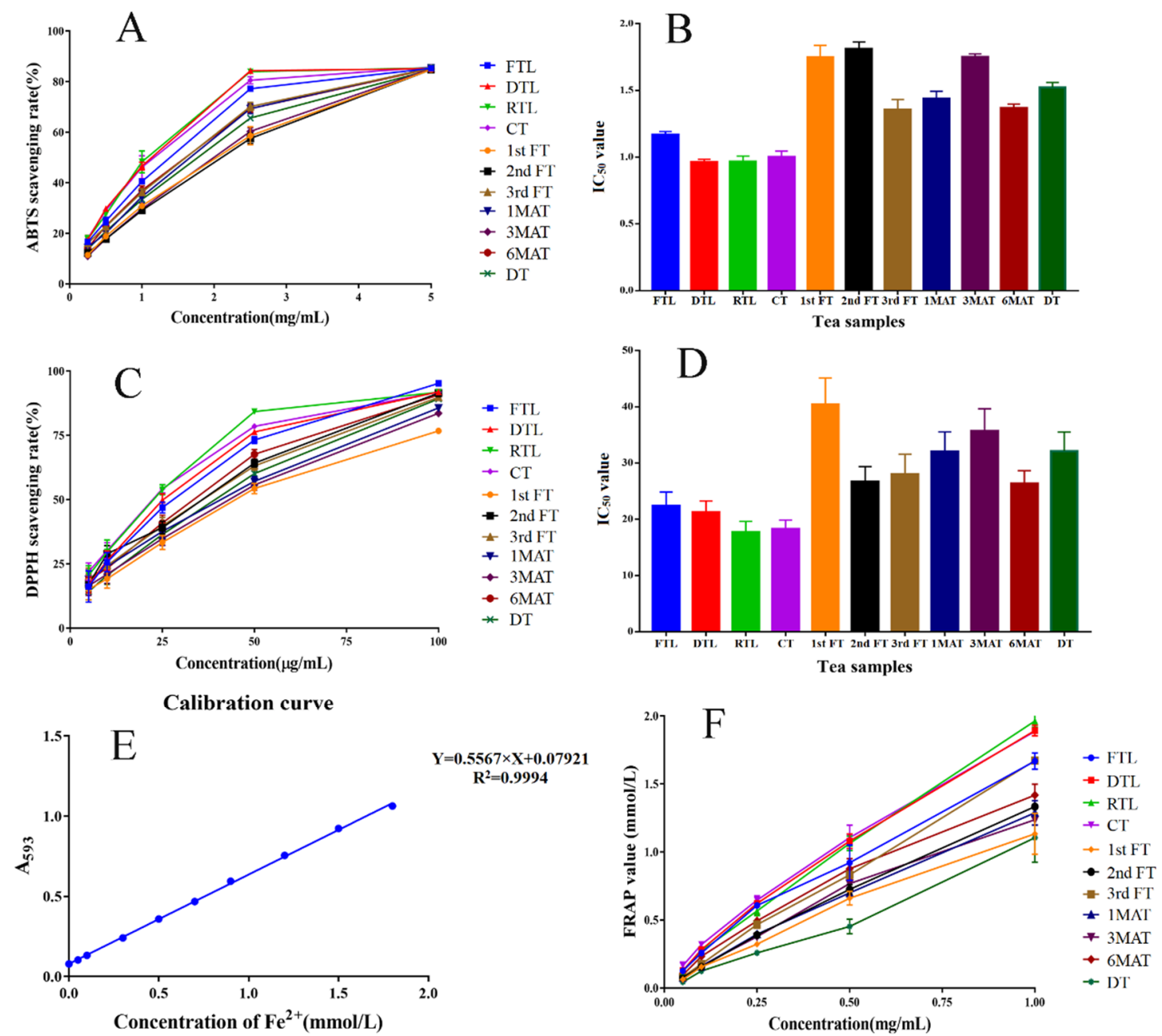

Figure 5. The antioxidant activity of different samples during QZT processing. (A) ABTS scavenging rates of different QZT samples. (B) $\mathrm{IC}_{50}$ values of different QZT samples on ABTS scavenging: FTL: $1.17 \mathrm{mg} / \mathrm{mL}$; DTL: $0.96 \mathrm{mg} / \mathrm{mL}$; RTL: $0.97 \mathrm{mg} / \mathrm{mL}$; CT: $1.00 \mathrm{mg} / \mathrm{mL}$; 1st FT: $1.75 \mathrm{mg} / \mathrm{mL}$; 2nd FT: $1.81 \mathrm{mg} / \mathrm{mL}$; 3rd FT: $1.36 \mathrm{mg} / \mathrm{mL}$; $1 \mathrm{MAT}: 1.44 \mathrm{mg} / \mathrm{mL} ; 3 \mathrm{MAT}$ : $1.75 \mathrm{mg} / \mathrm{mL}$; 6MAT: $1.37 \mathrm{mg} / \mathrm{mL}$; DT: $1.52 \mathrm{mg} / \mathrm{mL}$. (C) DPPH scavenging rates of different QZT samples. (D) $\mathrm{IC}_{50}$ values of different QZT samples on DPPH scavenging: FTL: $22.38 \mu \mathrm{g} / \mathrm{mL}$; DTL: $21.27 \mu \mathrm{g} / \mathrm{mL}$; RTL: 17.72 $\mu \mathrm{g} / \mathrm{mL}$; CT: 18.28 $\mu \mathrm{g} / \mathrm{mL}$; 1st FT: $40.43 \mu \mathrm{g} / \mathrm{mL}$; 2nd FT: $26.69 \mu \mathrm{g} / \mathrm{mL}$; 3rd FT: $27.99 \mu \mathrm{g} / \mathrm{mL}$; 1MAT: $32.03 \mu \mathrm{g} / \mathrm{mL}$; 3MAT: 35.71 $\mu \mathrm{g} / \mathrm{mL}$; 6MAT: $26.38 \mu \mathrm{g} / \mathrm{mL}$; DT: $32.14 \mu \mathrm{g} / \mathrm{mL}$. (E) The calibration curve of $\mathrm{Fe}^{2+}$. (F) FRAP values of different QZT samples with different concentrations $(0.05-1 \mathrm{mg} / \mathrm{mL})$.

\subsection{Correlation Analysis between Chemical Compositions and Biological Activities}

From the above results about chemical analysis and biological activities, including the antioxidant and inhibition effects on carbohydrate hydrolases, it can be seen the chemical compositions and biological activities of QZT samples were both varied during the processing. Polyphenols are usually considered the main bioactive compounds of tea [29]. In the present study, we also conducted a correlation analysis between metabolites and bioactivi- 
ties. As shown in Table 4, the total polyphenols and flavonoids were positively correlated to the antioxidant capacity and inhibitory effects on $\alpha$-amylase and $\alpha$-glucosidase. These results suggested the polyphenols were the main bioactive compounds of tea. Among all the polyphenols, the Pearson correlation coefficients of EGCG, GCG, ECG, EGC, and GC were greater than 0.5 . Other polyphenols, including quercetin-3-O-glucopyranosyl-Oglucopyranosyl-O-rhamnoside, myricetin-3-O-galactoside, and methoxy-epigallocatechin gallate, were also strongly related to tea's biological activities.

Table 4. Pearson correlation coefficients between chemical compositions and biological activities.

\begin{tabular}{|c|c|c|c|c|c|}
\hline \multirow{2}{*}{ Marker Compounds } & \multicolumn{5}{|c|}{ Pearson Correlation Coefficient } \\
\hline & ABTS & DPPH & FRAP & $\alpha$-Amylase & $\alpha$-Glucosidase \\
\hline Myricetin-3-O-galactoside & $0.719 *$ & $0.713 *$ & $0.799 * *$ & 0.552 & $0.724 *$ \\
\hline Methoxy-epigallocatechin gallate & $0.819 * *$ & $0.764^{* *}$ & $0.829 * *$ & 0.600 & $0.687 *$ \\
\hline Quercetin-3-O-glucopyranoside & $0.756^{* *}$ & $0.720 *$ & $0.785^{* *}$ & 0.561 & $0.750 * *$ \\
\hline Assamicain A/B/C & $0.832 * *$ & $0.765 * *$ & $0.817^{* *}$ & 0.586 & $0.661 *$ \\
\hline Methoxy-epicatechin gallate & $0.768^{* *}$ & $0.734 *$ & $0.789 * *$ & 0.595 & $0.738^{* *}$ \\
\hline L-theanine & $0.807^{* *}$ & $0.743^{* *}$ & $0.829 * *$ & 0.555 & $0.648 *$ \\
\hline Trihydroxyflavone-di-arabinopyranoside & $0.781^{* *}$ & $0.701 *$ & $0.776^{* *}$ & $0.602 *$ & $0.717 *$ \\
\hline 521.080 (unknown compound) & $0.828^{* *}$ & $0.763^{* *}$ & $0.791^{* *}$ & $0.615 *$ & $0.658 *$ \\
\hline Dihydrovomifoliol-O-glucopyranoside & $0.702 *$ & $0.645 *$ & $0.729 *$ & 0.580 & $0.781^{* *}$ \\
\hline Di-O-caffeoylquinic acid & $0.823^{* *}$ & $0.746^{* *}$ & $0.815^{* *}$ & 0.573 & $0.667 *$ \\
\hline 405.111 (unknown compound) & $0.760^{* *}$ & $0.680 *$ & $0.761^{* *}$ & 0.593 & $0.741^{* *}$ \\
\hline 307.077 (unknown compound) & $0.886^{* *}$ & $0.841^{* *}$ & $0.863^{* *}$ & $0.701 *$ & 0.512 \\
\hline 406.108 (unknown compound) & $0.793^{* *}$ & $0.721 *$ & $0.791^{* *}$ & $0.705 *$ & 0.543 \\
\hline Pentahydroxyflavanone & $0.889 * *$ & $0.840 * *$ & $0.854^{* *}$ & $0.745^{* *}$ & 0.423 \\
\hline 494.061 (unknown compound) & $0.793^{* *}$ & $0.733 *$ & $0.822^{* *}$ & 0.528 & $0.703 *$ \\
\hline 374.061 (unknown compound) & $0.880^{* *}$ & $0.830 * *$ & $0.834^{* *}$ & $0.713 *$ & 0.516 \\
\hline Theogallin & 0.108 & 0.075 & 0.242 & 0.045 & $0.764^{* *}$ \\
\hline 126.031 (unknown compound) & -0.469 & -0.431 & -0.522 & -0.304 & $-0.939 * *$ \\
\hline Junipetrioloside A & $0.862 * *$ & $0.795 * *$ & $0.854^{* *}$ & $0.616 *$ & 0.486 \\
\hline 548.054 (unknown compound) & $0.612 *$ & $0.658 *$ & $0.702 *$ & $0.640 *$ & $0.679 *$ \\
\hline$(-)$-epicatechin gallate-3"-O-Me & $0.791^{* *}$ & $0.750 * *$ & $0.811 * *$ & 0.598 & $0.720 *$ \\
\hline 182.079 (unknown compound) & -0.325 & -0.285 & -0.379 & -0.224 & $-0.965 * *$ \\
\hline Procyanidin B2 & $-0.704 *$ & -0.568 & -0.554 & -0.278 & -0.453 \\
\hline 938.150 (unknown compound) & $0.826^{* *}$ & $0.765 * *$ & $0.824^{* *}$ & 0.574 & $0.670 *$ \\
\hline 454.053 (unknown compound) & $0.750^{* *}$ & $0.740 * *$ & $0.783 * *$ & $0.637 *$ & $0.708 *$ \\
\hline 366.056 (unknown compound) & 0.447 & 0.534 & 0.552 & 0.393 & $0.806^{* *}$ \\
\hline 7-methoxy-kaempferol-glucopyranoside & 0.585 & 0.589 & $0.716 *$ & 0.411 & $0.751 * *$ \\
\hline $\begin{array}{l}\text { Quercetin 3-O-glucopyranosyl-O- } \\
\text { glucopyranosyl-O-rhamnoside }\end{array}$ & $0.772 * *$ & $0.711 *$ & $0.812 * *$ & 0.554 & $0.637 *$ \\
\hline Aqueous extracts & 0.55 & $0.633 *$ & $0.768^{* *}$ & 0.376 & 0.553 \\
\hline Total polyphenols & $0.620 *$ & 0.599 & $0.727 *$ & 0.479 & $0.736^{* *}$ \\
\hline Total amino acids & 0.592 & 0.566 & $0.717 *$ & 0.269 & $0.684 *$ \\
\hline Total flavonoids & $0.683 *$ & $0.638 *$ & $0.751 * *$ & 0.462 & $0.718 *$ \\
\hline Total soluble sugars & 0.364 & 0.351 & 0.485 & 0.350 & $0.688 *$ \\
\hline Gallic acid & -0.272 & -0.382 & -0.329 & -0.267 & $-0.748^{* *}$ \\
\hline Caffeine & 0.207 & 0.294 & 0.406 & 0.084 & 0.199 \\
\hline GC & 0.504 & 0.524 & $0.679 *$ & 0.204 & $0.753^{* *}$ \\
\hline EGC & 0.541 & 0.588 & $0.684 *$ & 0.447 & $0.689 *$ \\
\hline C & 0.354 & 0.314 & 0.524 & 0.274 & $0.653 *$ \\
\hline EGCG & 0.510 & 0.528 & $0.682 *$ & 0.445 & $0.714^{*}$ \\
\hline EC & 0.448 & 0.46 & $0.639 *$ & 0.477 & $0.684 *$ \\
\hline GCG & $0.624 *$ & $0.684 *$ & $0.754^{* *}$ & 0.382 & 0.626 * \\
\hline $\mathrm{ECG}$ & 0.531 & 0.53 & $0.661 *$ & 0.454 & $0.709 *$ \\
\hline $\mathrm{CG}$ & 0.277 & 0.075 & 0.314 & 0.210 & 0.373 \\
\hline Total catechins & 0.535 & 0.555 & $0.698 *$ & 0.421 & $0.718 *$ \\
\hline
\end{tabular}




\section{Conclusions}

Pile-fermentation had stronger effects on chemical compositions and biological activities, including antioxidant capacities and inhibitory effects on $\alpha$-amylase and $\alpha$-glucosidase. QZT usually needs long-term processing, especially for pile-fermentation and aging, whereas the biological activities of tea samples were reduced during the processing with the decreasing of polyphenols. Galloylated catechins and flavonoids glycosides were also degraded after pile-fermentation, which is similar to the results of ripened Pu-erh tea during post-fermentation. However, the loss of catechins and flavonol glycosides, which mainly belong to the astringent and bitter compounds, will contribute to the improvement of sensory quality of QZT and hence increase the consumer acceptance. The present study indicated that the flavor (especially the astringent taste) of the mature tea leaves was markedly improved after long-term pile-fermentation and aging, whereas its biological activities were correspondingly decreased. Therefore, the fermentation and aging processing time of QZT need be controlled and optimized.

Author Contributions: Conceptualization, P.-C.Z. and C.-Y.Q.; Methodology, P.-C.Z., C.-Y.Q., and P.-P.L.; Software, P.-C.Z., C.-Y.Q., and P.-P.L.; Validation, J.-M.N.; Formal Analysis, P.-C.Z., C.-Y.Q., and P.-P.L.; Investigation, L.F. and T.-J.L.; Resources, X.-C.W. and L.Z.; Data Curation, P.-C.Z. and C.-Y.Q.; Writing-Original Draft Preparation, P.-C.Z. and C.-Y.Q.; Writing-Review \& Editing, J.M.N.; Visualization, L.F. and T.-J.L.; Supervision, T.-J.L. and J.-M.N.; Project Administration, X.-C.W. and L.Z.; Funding Acquisition, X.-C.W. and L.Z. All authors have read and agreed to the published version of the manuscript.

Funding: This work was funded by Natural Science Foundation of China (32072633, 32072634, 31902081), earmarked fund for China Agriculture Research System of MOF and MARA (CARS-19), Anhui Key research and development plan (202104b11020001, 1804b06020367, 202004b11020004), and Young Elite Scientist Sponsorship Program by National CAST (2016QNRC001).

Institutional Review Board Statement: Not applicable.

Informed Consent Statement: Not applicable.

Data Availability Statement: Not available.

Conflicts of Interest: The authors declare no conflict of interest.

Samples Availability: Samples of the compounds Gallic acid, caffeine, theobromine, $(+)$ — catechin, $(-)$ epicatechin, (-) — gallocatechin, (-) —epigallocatechin, (-) — gallocatechin gallate, $(-)$ —epigallocatechin gallate, and (-) - epicatechin gallate are available from the authors.

\section{References}

1. Cheng, Q.; Cai, S.; Ni, D.; Wang, R.; Zhou, F.; Ji, B.; Chen, Y. In vitro antioxidant and pancreatic $\alpha$-amylase inhibitory activity of isolated fractions from water extract of Qingzhuan tea. J. Food Sci. Technol. 2013, 52, 928-935. [CrossRef] [PubMed]

2. Yang, X.; Huang, M.; Qin, C.; Lv, B.; Mao, Q.; Liu, Z. Structural characterization and evaluation of the antioxidant activities of polysaccharides extracted from Qingzhuan brick tea. Int. J. Biol. Macromol. 2017, 101, 768-775. [CrossRef]

3. Zhang, L.; Ho, C.; Zhou, J.; Santos, J.S.; Armstrong, L.; Granato, D. Chemistry and Biological Activities of Processed Camellia sinensis Teas: A Comprehensive Review. Compr. Rev. Food Sci. Food Saf. 2019, 18, 1474-1495. [CrossRef] [PubMed]

4. $\quad$ Cheng, L.; Yang, Q.-Q.; Chen, Z.; Zhang, J.-R.; Chen, Q.; Wang, Y.; Wei, X. Distinct Changes of Metabolic Profile and Sensory Quality during Qingzhuan Tea Processing Revealed by LC-MS-Based Metabolomics. J. Agric. Food Chem. 2020, 68, $4955-4965$. [CrossRef] [PubMed]

5. Zheng, W.-J.; Wan, X.-C.; Bao, G.-H. Brick dark tea: A review of the manufacture, chemical constituents and bioconversion of the major chemical components during fermentation. Phytochem. Rev. 2015, 14, 499-523. [CrossRef]

6. Feng, L.; Zhou, J.; Zhang, L.; Liu, P.; Zheng, P.; Gao, S.; Song, C.; Yu, Y.; Gong, Z.; Wan, X. Gut microbiota-mediated improvement of metabolic disorders by Qingzhuan tea in high fat diet-fed mice. J. Funct. Foods 2021, 78, 104366. [CrossRef]

7. Li, P.; Dai, W.; Lu, M.; Xie, D.; Tan, J.; Yang, C.; Zhu, Y.; Lv, H.; Peng, Q.; Zhang, Y.; et al. Metabolomic analysis reveals the composition differences in 13 Chinese tea cultivars of different manufacturing suitabilities. J. Sci. Food Agric. 2017, 98, 1153-1161. [CrossRef] [PubMed]

8. Zhang, L.; Deng, W.-W.; Wan, X.-C. Advantage of LC-MS metabolomics to identify marker compounds in two types of Chinese dark tea after different post-fermentation processes. Food Sci. Biotechnol. 2014, 23, 355-360. [CrossRef] 
9. Zhang, L.; Zhang, Z.-Z.; Zhou, Y.-B.; Ling, T.-J.; Wan, X.-C. Chinese dark teas: Postfermentation, chemistry and biological activities. Food Res. Int. 2013, 53, 600-607. [CrossRef]

10. Zhou, J.; Wu, Y.; Long, P.; Ho, C.-T.; Wang, Y.; Kan, Z.; Cao, L.; Zhang, L.; Wan, X. LC-MS-Based Metabolomics Reveals the Chemical Changes of Polyphenols during High-Temperature Roasting of Large-Leaf Yellow Tea. J. Agric. Food Chem. 2019, 67, 5405-5412. [CrossRef]

11. Long, P.; Wen, M.; Granato, D.; Zhou, J.; Wu, Y.; Hou, Y.; Zhang, L. Untargeted and targeted metabolomics reveal the chemical characteristic of pu-erh tea (Camellia assamica) during pile-fermentation. Food Chem. 2019, 311, 125895. [CrossRef] [PubMed]

12. Shalini, V.; Hussain, M.E. Obesity and diabetes: An update. Diabetes Metab. Syndr. 2017, 11, 73-79.

13. Cai, X.; Liu, Z.; Dong, X.; Wang, Y.; Zhu, L.; Li, M.; Xu, Y. Hypoglycemic and lipid lowering effects of theaflavins in high-fat diet-induced obese mice. Food Funct. 2021, 12, 9922-9931. [CrossRef] [PubMed]

14. Falla, N.; Demasi, S.; Caser, M.; Scariot, V. Phytochemical Profile and Antioxidant Properties of Italian Green Tea, a New High Quality Niche Product. Horticulturae 2021, 7, 91. [CrossRef]

15. Liu, C.; Guo, Y.; Sun, L.; Lai, X.; Li, Q.; Zhang, W.; Xiang, L.; Sun, S.; Cao, F. Six types of tea reduce high-fat-diet-induced fat accumulation in mice by increasing lipid metabolism and suppressing inflammation. Food Funct. 2019, 10, 2061-2074. [CrossRef] [PubMed]

16. Roda, G.; Marinello, C.; Grassi, A.; Picozzi, C.; Aldini, G.; Carini, M.; Regazzoni, L. Ripe and Raw Pu-Erh Tea: LC-MS Profiling, Antioxidant Capacity and Enzyme Inhibition Activities of Aqueous and Hydro-Alcoholic Extracts. Molecules 2019, 24, 473. [CrossRef]

17. Feng, L.; Liu, P.; Zheng, P.; Zhang, L.; Zhou, J.; Gong, Z.; Yu, Y.; Gao, S.; Zheng, L.; Wang, X.; et al. Chemical profile changes during pile fermentation of Qingzhuan tea affect inhibition of $\alpha$-amylase and lipase. Sci. Rep. 2020, 10, 1-10. [CrossRef]

18. Lin, F.-J.; Wei, X.-L.; Liu, H.-Y.; Li, H.; Xia, Y.; Wu, D.-T.; Zhang, P.-Z.; Gandhi, G.R.; Li, H.-B.; Gan, R.-Y. State-of-the-art review of dark tea: From chemistry to health benefits. Trends Food Sci. Technol. 2021, 109, 126-138. [CrossRef]

19. Zhu, M.; Li, N.; Zhou, F.; Ouyang, J.; Lu, D.-M.; Xu, W.; Li, J.; Lin, H.-Y.; Zhang, Z.; Xiao, J.; et al. Microbial bioconversion of the chemical components in dark tea. Food Chem. 2019, 312, 126043. [CrossRef]

20. Guo, X.; Long, P.; Meng, Q.; Ho, C.-T.; Zhang, L. An emerging strategy for evaluating the grades of Keemun black tea by combinatory liquid chromatography-Orbitrap mass spectrometry-based untargeted metabolomics and inhibition effects on $\alpha$-glucosidase and $\alpha$-amylase. Food Chem. 2018, 246, 74-81. [CrossRef] [PubMed]

21. Benzie, I.F.F.; Strain, J.J. Simultaneous automated measurement of total 'antioxidant' (reducing) capacity and ascorbic acid concentration. Redox Rep. 1997, 3, 233-238. [CrossRef] [PubMed]

22. Brand-Williams, W.; Cuvelier, M.; Berset, C. Use of a free radical method to evaluate antioxidant activity. LWT 1995, 28, 25-30. [CrossRef]

23. Zhang, L.; Santos, J.S.; Cruz, T.M.; Marques, M.B.; Carmo, M.A.V.D.; Azevedo, L.; Wang, Y.; Granato, D. Multivariate effects of Chinese keemun black tea grades (Camellia sinensis var. sinensis) on the phenolic composition, antioxidant, antihemolytic and cytotoxic/cytoprotection activities. Food Res. Int. 2019, 125, 108516. [CrossRef] [PubMed]

24. Cheng, L.; Wang, Y.; Zhang, J.; Xu, L.; Zhou, H.; Wei, K.; Peng, L.; Zhang, J.; Liu, Z.; Wei, X. Integration of non-targeted metabolomics and E-tongue evaluation reveals the chemical variation and taste characteristics of five typical dark teas. $L W T$ 2021, 150, 111875. [CrossRef]

25. Li, Q.; Jin, Y.; Jiang, R.; Xu, Y.; Zhang, Y.; Luo, Y.; Huang, J.; Wang, K.; Liu, Z. Dynamic changes in the metabolite profile and taste characteristics of Fu brick tea during the manufacturing process. Food Chem. 2020, 344, 128576. [CrossRef] [PubMed]

26. Lv, H.-P.; Zhang, Y.-J.; Lin, Z.; Liang, Y.-R. Processing and chemical constituents of Pu-erh tea: A review. Food Res. Int. 2013, 53, 608-618. [CrossRef]

27. Wang, Z.; Zheng, C.; Ma, C.; Ma, B.; Wang, J.; Zhou, B.; Xia, T. Comparative analysis of chemical constituents and antioxidant activity in tea-leaves microbial fermentation of seven tea-derived fungi from ripened Pu-erh tea. LWT 2021, 142, 111006. [CrossRef]

28. Lv, H.-P.; Zhang, Y.; Shi, J.; Lin, Z. Phytochemical profiles and antioxidant activities of Chinese dark teas obtained by different processing technologies. Food Res. Int. 2017, 100, 486-493. [CrossRef]

29. Chen, Y.; Cheng, S.; Dai, J.; Wang, L.; Xu, Y.; Peng, X.; Xie, X.; Peng, C. Molecular mechanisms and applications of tea polyphenols: A narrative review. J. Food Biochem. 2021, 45, e13910. [CrossRef] 